\title{
ON THE ANTITONE MAPPINGS OF A POSET ${ }^{1}$
}

\section{F. JANOWITZ}

1. Introduction. Following the terminology in $[3$, p. 70$]$, let us agree first of all that by an involution poset $(L, ')$, we will mean the following: $L$ is a partially ordered set with a largest element 1 equipped with a mapping ': $L \rightarrow L$ called the involution such that for $e, f \in L, e \leqq f \Rightarrow f^{\prime} \leqq e^{\prime}$, and for $e \in L, e=e^{\prime \prime}$. If also (i) for $e, f \in L$ with $e \leqq f^{\prime}, \sup \{e, f\}=e \bigvee f$ exists, and (ii) for $e, f \in L, e \leqq f \Rightarrow f=e \bigvee\left(f^{\prime} \bigvee e\right)^{\prime}$, then $L$ will be called an orthomodular poset. Finally, we define an orthomodular lattice to be an orthomodular poset that is also a lattice.

In [4, pp. 96-107], G. Mackey presents an argument that shows on quite plausible physical grounds that the "logic" of (nonrelativistic) quantum mechanics forms an orthomodular poset. He then assumes on grounds of "regularity" that it is actually an orthomodular lattice, in fact that it is the lattice of closed subspaces of a separable infinite-dimensional Hilbert space. It would be nice to find additional physical justification for this last assumption, and therefore any condition on an involution poset which guarantees that it is actually an orthomodular lattice has importance.

The following approach to this question has recently been developed by $D$. J. Foulis [3, pp. 70-72]. Assuming $\left(L,{ }^{\prime}\right)$ to be an involution poset, let $S(L)$ denote the set of all monotone maps $\phi: L \rightarrow L$ having the property that there exists a (necessarily unique) monotone map $\phi^{*}: L \rightarrow L$ such that $(e \phi)^{\prime} \phi^{*} \leqq e^{\prime}$ and $\left(e \phi^{*}\right)^{\prime} \phi \leqq e^{\prime}$ for each $e \in L$. An element $\phi \in S(L)$ is called a *-monotone mapping, and the element $\phi^{*}$ is known as the adjoint of $\phi$. Foulis [2, p. 648] defines an involution semigroup to be a multiplicatively written semigroup $S$ equipped with a mapping *: $S \rightarrow S$, (called the involution), such that for $x, y \in S$, $(x y)^{*}=y^{*} x^{*}$ and $\left(x^{*}\right)^{*}=x^{* *}=x$. It is easily verified that $S(L)$ provides an example of an involution semigroup with a zero element (having function composition as the semigroup operation and $\phi \rightarrow \phi^{*}$ as the involution).

A semigroup $S$ is called a Baer *-semigroup in case it is an involution semigroup with a zero element 0 such that for each $x \in S$ there

Received by the editors April 23, 1963.

1 The results in this paper form part of the author's doctoral dissertation (Wayne State University, 1963) written under the direction of Professor D. J. Foulis. The research was supported by a National Science Foundation Summer Fellowship, and by a National Science Foundation Cooperative Graduate Fellowship. 
exists a (necessarily unique) element $x^{\prime} \in S$ with $\{y \in S: x y=0\}$ $=x^{\prime} S$, and $x^{\prime}=\left(x^{\prime}\right)^{2}=\left(x^{\prime}\right)^{*}$, where ${ }^{*}$ is the involution mapping in $S$. We then have the following result whose proof is given in $[3$, Theorem 10, p. 71$]$.

THEOREM 1. The necessary and sufficient condition that the involution poset $\left(L,{ }^{\prime}\right)$ be an orthomodular lattice is that the involution semigroup $S(L)$ be a Baer *-semigroup.

By considering antitone rather than monotone mappings, we shall generalize this result by obtaining necessary and sufficient conditions for an antitone mapping on an arbitrary poset $L$ with 0 and 1 to turn $L$ into an orthomodular lattice. The author wishes to point out that many of his ideas were obtained from a consideration of results in [3] and [5]. He also wishes to thank Professor D. J. Foulis for directing the research necessary for this paper.

2. Antitone mappings. In this section $L$ and $M$ will denote partially ordered sets having a largest element 1 and a smallest element 0 . Letters in the first half of the alphabet will denote elements of $L$, while those of the second half will be reserved for elements of $M$. The symbols 1 and 0 will ambiguously denote the largest and smallest elements of both $L$ and $M$.

By an antitone mapping of $L$ into $M$, we shall mean a mapping $\alpha: L \rightarrow M$ such that for $e, f \in L, e \leqq f \Rightarrow f \alpha \leqq e \alpha$. If $\alpha: L \rightarrow M$ and $\beta: M \rightarrow L$ are each antitone mappings, then the pair $(\alpha, \beta)$ is said to form a Galois connection in case for each $e \in L, x \in M$, we have $e \leqq e \alpha \beta$ and $x \leqq x \beta \alpha$.

LEMmA 2. If $\alpha: L \rightarrow M, \beta: M \rightarrow L, \gamma: M \rightarrow L$ are antitone mappings having the property that $(\alpha, \beta)$ and $(\alpha, \gamma)$ each form Galois connections, then $\beta=\gamma$.

Proof. Let $x \in M$. Then $x \leqq x \beta \alpha$, so that $x \beta \alpha \gamma \leqq x \gamma$. Hence $x \beta$ $\leqq x \beta \alpha \gamma \leqq x \gamma$; similarly, $x \gamma \leqq x \beta$.

This shows that an antitone mapping $\alpha: L \rightarrow M$ can form a Galois connection with at most one antitone mapping $\beta: M \rightarrow L$. For reasons that will soon be apparent, such a mapping $\beta: M \rightarrow L$ (if it exists) will be called the adjoint of $\alpha$ and frequently indicated by the symbol $\alpha^{*}$. The set of all antitone mappings from $L$ into $M$ possessing adjoints will be denoted by $G(L, M)$; if $L=M$, we shall write $G(L)$ in place of $G(L, L)$.

LemMa 3. For each $x \in M$, there is a mapping $\alpha \in G(L, M)$ such that $1 \alpha=x$. 
Proof. Define mappings $\alpha: L \rightarrow M$ and $\beta: M \rightarrow L$ as follows, and then compute: For $e \in L$, let $e \alpha=x$ if $e \neq 0$, and $0 \alpha=1$. For $y \in M$, let $y \beta=1$ if $y \leqq x$ and $y \beta=0$ otherwise.

For the case where $L=M$, since the composition of two antitone mappings is monotone, it is pointless to try making $G(L)$ into a semigroup with respect to function composition. However, the composition of three antitone mappings is again antitone, so that we are led to the following operation on $G(L)$ : Let $\# \in G(L)$ have + as its adjoint. Given $\alpha, \beta \in G(L)$, define $\alpha \odot \beta$ by the formula $e(\alpha \odot \beta)$ $=e \alpha \# \beta$ for each $e \in L$. Naturally enough, we now wonder just when $(G(L), \odot)$ is a semigroup, an involution semigroup, or better still a Baer *-semigroup. Note that when we speak of $(G(L), \odot)$ as being an involution semigroup, we shall always be referring to the mapping $\alpha \rightarrow \alpha^{*}$ as the involution.

Lemma 4. For arbitrary $e \in L, e \#=e \#+\#$ and $e+=e+\#+$.

Proof. See [1, p. 56].

Lemma 5. The necessary and sufficient condition that $(G(L), \odot)$ be an involution semigroup is that e\#\# $\leqq$ for each $e \in L$.

Proof. (i) Assume $(G(L), \odot)$ is an involution semigroup. Define mappings $\alpha, \beta: L \rightarrow L$ as follows: Choose an arbitrary element $e \in L$. Set $g \alpha=e$ if $g \neq 0,0 \alpha=1 ; g \beta=1$ if $g \leqq e, g \beta=0$ otherwise. From the proof of Lemma 3, we see that $\alpha \in G(L)$ with $\beta=\alpha^{*}$. Let $\gamma=\alpha \odot \#$; by assumption $\gamma^{*}=+\odot \alpha^{*}=+\odot \beta$, hence $1 \leqq 1 \gamma \gamma^{*}=1(\alpha \odot \#)(+\odot \beta)$ $=1 \alpha \# \#+\# \beta=e \#(\#+\#) \beta=e \# \# \beta$. Thus $e \# \# \beta=1$, and by the definition of $\beta$, we have that $e \# \# \leqq e$.

(ii) Suppose conversely that $e \# \# \leqq e$ for every $e \in L$. Given $\alpha, \beta$ $\in G(L)$, we are to show that $\alpha \odot \beta \in G(L)$ with $(\alpha \odot \beta)^{*}=\beta^{*} \odot \alpha^{*}$; i.e., that for each $e \in L, e \leqq e \alpha \# \beta \beta^{*} \# \alpha^{*}$ and $e \leqq e \beta^{*} \# \alpha^{*} \alpha \# \beta$. We know that $g \leqq g \beta \beta^{*}$ for any $g \in L$, and in particular that $e \alpha \# \leqq e \alpha \# \beta \beta^{*}$. Applying $\#$ and then $\alpha^{*}$ to this inequality produces $e \alpha \# \# \alpha^{*} \leqq e \alpha \# \beta \beta^{*} \# \alpha^{*}$. But $(e \alpha) \# \# \leqq e \alpha$ implies that $e \leqq e \alpha \alpha^{*} \leqq e \alpha \# \# \alpha^{*} \leqq e \alpha \# \beta \beta^{*} \# \alpha^{*}$. Similarly, $e \leqq e \beta^{*} \# \alpha^{*} \alpha \# \beta$.

Lemma 6. $(G(L), \odot)$ is an involution semigroup with a multiplicative identity if and only if $(L, \#)$ is an involution poset.

Proof. (i) Suppose that $(G(L), \odot)$ is an involution semigroup having $\iota$ as a multiplicative identity. Then $\iota \odot+=+\odot \iota=+$, so that for any $e \in L, e+=e$ ı\# $+=e+\# \iota$. Applying Lemma 4, we see that

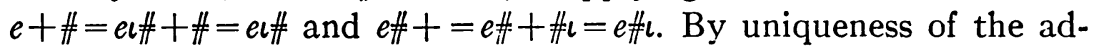
joint of \# it follows that $+=\imath$. By Lemma 5 , $e \# \# \leqq e$ for every $e \in L$, 
and therefore $e \# \leqq e \# \#$. Clearly $e \# \# \#=(e \#) \# \# \leqq e \#$, whence $e \#=e \# \# \#$ for arbitrary $e \in L$. Then $e \#=e(+\odot \#)=e+\# \#, e \# \#=e+\# \#=e+\#$, and finally, $e \leqq e+\#=e \# \#$. Therefore $e \# \#=e$, and $(L, \#)$ is indeed an involution poset.

(ii) If $(L, \#)$ is an involution poset, then by Lemma $5,(G(L), \odot)$ is an involution semigroup. The involution \# is obviously effective as the multiplicative identity of $G(L)$.

Lemma 7. If $(L, \#)$ is an involution poset, then there is an involution preserving semigroup isomorphism between $S(L)$ and $(G(L), \odot)$.

Proof. If $\phi \in S(L)$, let $\alpha(\phi)=\phi \#$. Then $\alpha(\phi)$ is antitone, and since $e \phi \# \phi^{*} \leqq e \#$, we have $e \leqq e \phi \# \phi^{*} \#=e \alpha(\phi) \alpha\left(\phi^{*}\right)$. Similarly, $e \leqq e \alpha\left(\phi^{*}\right) \alpha(\phi)$, and therefore $\alpha(\phi) \in G(L)$ with $[\alpha(\phi)]^{*}=\alpha\left(\phi^{*}\right)$. If $\alpha \in G(L)$, define $\phi(\alpha)$ by $\phi(\alpha)=\alpha \#$. Then $\phi(\alpha)$ is monotone, and $e \leqq e \alpha \alpha^{*}$ implies that $e \alpha \# \# \alpha^{*} \#=e \alpha \alpha^{*} \# \leqq e \#$;i.e., $[e \phi(\alpha)] \# \phi\left(\alpha^{*}\right) \leqq e \#$.Similarly, $\left[e \phi\left(\alpha^{*}\right)\right] \# \phi(\alpha)$ $\leqq e \#$, so that $\phi(\alpha) \in S(L)$ with $[\phi(\alpha)]^{*}=\phi\left(\alpha^{*}\right)$. In view of the involutory nature of \# it is evident that $\phi=\phi(\alpha(\phi))$ and $\alpha=\alpha(\phi(\alpha))$, so that we have set up a one-one involution preserving correspondence between $S(L)$ and $(G(L), \odot)$. The proof is completed by noting that for $\phi, \psi \in S(L), \alpha, \beta \in G(L)$, and $e \in L, e \alpha(\phi \psi)=e \phi \psi \#=e \phi \# \# \psi \#$ $=e(\alpha(\phi) \odot \alpha(\psi))$, and $e \phi(\alpha \odot \beta)=e \alpha \# \beta \#=e \phi(\alpha) \phi(\beta)$.

We are now ready to produce a necessary and sufficient condition in terms of antitone mappings for a poset with 0 and 1 to be an orthomodular lattice.

Theorem 8. Let $L$ be a poset with 0 and 1 . Choose a fixed element \# of $G(L)$, and for $\alpha, \beta \in G(L)$, let $\alpha \odot \beta=\alpha \# \beta$. Then, the following conditions are mutually equivalent:

(i) $(L, \#)$ is an orthomodular lattice.

(ii) $(L, \#)$ is an involution poset and $S(L)$ is a Baer *-semigroup.

(iii) $(G(L), \odot)$ is a Baer *-semigroup.

Proof (i) $\Leftrightarrow($ ii). This is a consequence of Theorem 1 .

(ii) $\Rightarrow$ (iii). By Lemma 7 there is an involution preserving semigroup isomorphism between $S(L)$ and $(G(L), \odot)$. Hence $(G(L), \odot)$ is a Baer *-semigroup.

(iii) $\Rightarrow$ (ii). If $(G(L), \odot)$ is to be a Baer *-semigroup, then it must first of all be an involution semigroup with identity. By Lemma 6, $(L, \#)$ is an involution poset, and making use of Lemma 7 , we see that $S(L)$ is a Baer *-semigroup.

3. Change of involution. There is one final question we shall consider here. Suppose that $(L, \#)$ is an involution poset, and that + is another involution on $L$. The set $G(L)$ is independent of the choice 
of involution, while the definition of $S(L)$ depends on the particular involution used. Conceivably, distinct involutions could lead to distinct sets of the form $S(L)$. It turns out, however, that a change in the involution on $L$ corresponds to a change in the notion of adjoint, but leaves the elements of $S(L)$ fixed. We adopt the following temporary notation: Let $S(L$, \#) denote the set of *-monotone mappings of $(L, \#)$, and for $\phi \in S(L, \#)$, let $\phi_{\|}^{*}$ denote its adjoint as computed in $S(L, \#)$. Analogous notation applies for $(L,+)$.

THEOREM 9. Let $(L, \#)$ be an involution poset. If + is another involution on $L$, then $S(L, \#)=S(L,+)$. Furthermore, there exists an element $\psi \in S(L, \#)$ such that: (i) $\psi=\psi_{\#}^{*}$; (ii) $+=\psi \#$; (iii) $\psi^{-1}=\# \psi \#$; (iv) for each $\phi \in S(L, \#), \phi_{+}^{*}=\psi \phi_{*}^{*} \psi^{-1}$. In fact, there is a one-one correspondence between changes of involution and mappings $\psi \in S(L, \#)$ with $\psi^{-1}=\# \psi \#$.

Proof. (a) Since + is clearly in $G(L)$, Lemma 7 shows that $+\# \in S(L, \#)$, and we may set $\psi=+\#$. It is obvious that $\psi^{-1}=\#+$ $=\#+\# \#=\# \psi \#$, and since $(e \psi) \# \psi=(e+\#) \#+\#=e++\#=e \#$, we have $\psi_{\#}^{*}=\psi$. Note that if $\phi \in S(L, \#)$, then so is $\phi \psi=\phi+\#$. This shows that $\phi+=(\phi+\#) \# G(L)$, and finally that $\phi=(\phi+)+\in S(L,+)$. Thus $S(L, \#) \subset S(L,+)$, and the reverse inclusion is obtained by interchanging the roles of \# and + in the above argument. It is now unambiguous to write $S(L)$ in place of $S(L, \#)$ or $S(L,+)$. Given $\phi \in S(L)$, we must determine $\phi_{+}^{*}$. To do this it suffices to find an $\alpha \in G(L)$ such that $(\phi+, \alpha)$ forms a Galois connection. This is equivalent to saying that $\alpha \#=(\phi+\#)_{\sharp}^{*}=(\phi \psi)_{\sharp}^{*}=\psi \phi_{\#}^{*}$. It follows that

$$
\phi_{+}^{*}=\alpha+=\alpha \# \#+=\psi \phi_{\#}^{*} \#+=\psi \phi_{\#}^{*} \psi^{-1} .
$$

(b) Let $\psi \in S(L)$ with $\psi^{-1}=\# \psi \#$, and set $+=\psi \#$. Then + is clearly antitone, and for $e \in L, e++=e \psi \# \psi \#=e \psi \psi^{-1}=e$, so that + is an involution on $L$. Thus each $\psi \in S(L)$ with the property that $\psi^{-1}=\# \psi \#$ gives rise to an involution on $L$, and (a) shows that every involution arises in this manner. To show that the correspondence is one-one, we need only note that if $+=\psi \#$, then $\psi=+\#$.

\section{BibliogRAPHY}

1. G. Birkhoff, Lattice theory, Amer. Math. Soc. Colloq. Publ. Vol. 25, Amer. Math. Soc., Providence, R. I., 1948.

2. D. J. Foulis, Baer *-semigroups, Proc. Amer. Math. Soc. 11 (1960), 648-654.

3. - A note on orthomodular lattices, Portugal. Math. 21 (1962), 65-72.

4. G. W. Mackey, Lecture notes on quantum mechanics, Harvard Univ., Cambridge, Mass., 1960.

5. F. B. Wright, Polarity and duality, Pacific J. Math. 10 (1960), 723-730.

Wayne State University 